\title{
The Functional Anatomy of Inhibition Processes Investigated with the Hayling Task
}

\author{
F. Collette, ${ }^{*}$ M. Van der Linden, ${ }^{*}$ G. Delfiore, $\dagger$ C. Degueldre, $\dagger$ A. Luxen, $\dagger$ and E. Salmon $\dagger$, $\ddagger$ \\ * Department of Neuropsychology, \\ $\dagger$ Cyclotron Research Centre, and \\ † Department of Neurology, University of Liège, Belgium
}

\begin{abstract}
The cortical areas involved in inhibition processes were examined with positron emission tomography (PET). The tasks administered to subjects were an adaptation of the Hayling test. In the first condition (response initiation), subjects had to complete sentences with a word clearly suggested by the context, whereas in the second condition (response inhibition), subjects had to produce a word that made no sense in the context of the sentence. Results indicated that the response initiation processes were associated to increases of activity in the left inferior frontal gyrus (BA 45/47), whereas response inhibition processes led to increases in a network of left prefrontal areas, including the middle (BA 9 and BA 10) and inferior (BA 45) frontal areas.
\end{abstract}

Key Words: positron emission tomography; inhibition; executive functions; prefrontal cortex.

\section{INTRODUCTION}

It is generally considered that inhibitory control constitutes an important executive function (Baddeley, 1986; Norman and Shallice, 1986). Different aspects of inhibitory control may be distinguished (Hasher et al., 1999), especially preventing access to goal-irrelevant information that may be partially activated, restraining access to strong but situationally inappropriate responses, and suppressing the activation of no-longer relevant information. Numerous clinical observations and experimental evidence have suggested that the prefrontal cortex plays a central role in the process of inhibitory control. For example, it has been shown that lesions to the frontal lobes may lead to perseverative tendencies and increased susceptibility to interference effects in various cognitive tasks such as the Wisconsin Card Sorting Test, (WCST, Milner, 1964), the Stroop test (Perret, 1974), the Tower of Hanoi (Glosser and Goodglass, 1990), tests of mental fluency (Stuss et al., 1998), the AB-AC paired-associate learning task (Shimamura et al., 1995), the Go-No-Go test (Leimkuhler and Mesulam, 1985), the negative priming task (Metzler and Parkin, 2000), or the Hayling test (Burgess and Shallice, 1996).

However, other studies did not reveal impaired performance or showed very little impairment on inhibition tasks in patients with focal frontal damage (Ahola et al., 1996; Anderson et al., 1991; Andrès and Van der Linden, 2000), and there is also evidence suggesting that patients with nonfrontal lesions can show interference and perseveration deficits similar to frontal patients (e.g., Mountain and Snow-William, 1993).

Another way to explore the neural substrate of inhibition control is functional imagery in normal subjects. Most of the studies in this domain used different variants of the Stroop paradigm and showed increased activity during the interference condition (i.e., naming the font colour of letters that spell a colour word different than the colorto-be-named) in the anterior cingulate gyrus and right orbitofrontal regions (Bench et al., 1993; Larrue et al., 1994; Pardo et al., 1990). Moreover, increased activity has also been found in left inferior frontal regions (Busch et al., 1998; George et al., 1994; Taylor et al., 1997) and in temporal and parietal areas (e.g., Bush et al., 1998; Taylor et al., 1997). More recently, Konishi et al. (1999) explored cerebral areas associated in inhibition processes by using two different cognitive tasks: the go/no-go paradigm and the Wisconsin card sorting test (WCST). Common transient activation related to inhibition of the go response and set shifting in the WCST was found in the right inferior prefrontal area (BA 45), leading to the conclusion that this prefrontal area is commonly involved in the inhibition of different targets.

Finally, Nathaniel-James et al. (1997) also explored the inhibition processes involved in the Hayling task. In their study, subjects were visually presented sentences with the last word missing. In one condition, they had to complete the sentence with the expected word ("initiation"), while in the other condition they had to provide a word unrelated to the sentence ("inhibition"). The modifications of metabolism in these two conditions were also compared to a condition in which subjects had to read the last word of the sentence. In comparison to the reading 
condition, both the initiation and inhibition conditions led to increased $\mathrm{rCBF}$ in the left operculum (BA 45) and right cingulate area (BA 32). However, when the inhibition condition was compared to the initiation condition, no supplementary activation focus was found. A possible interpretation of that absence of supplementary activity relates to the paradigm used. Indeed, the procedure of Nathaniel-James et al. could be insensitive to inhibition processes since sentences were presented every $6 \mathrm{~s}$ for each condition. Thus, if subjects gave responses faster than the time limit of $6 \mathrm{~s}$, they had some extra time in each condition during which cognitive processes were not controlled. This procedure could have made the task less sensitive to inhibition processes since these processes are not maximised in the contrast between the inhibition and initiation condition.

The aim of the present study was to reexamine the cerebral areas involved in the initiation and inhibition conditions of the Hayling task. The procedure used by Nathaniel-James et al. (1997) was modified in order to maximise the involvement of inhibition processes: for all conditions, the next sentence was displayed as soon as the subjects produced a response. Moreover, subjects were trained before the experimental session to use inhibition processes efficiently.

\section{MATERIAL AND METHODS}

\section{Subjects}

Twelve European right-handed volunteers (six males and six females, age range from 21 to 28 years), gave written informed consent to take part in this study, which was approved by the University of Lie'ge Ethics Committee. None had any past medical history nor used any medication.

\section{Positron Emission Tomography Scanning}

PET data were acquired on a Siemens CTI 951 R 16/31 scanner in 3-D mode. The subject's head was stabilized by a thermoplastic facemask secured to the head holder (Truscan imaging, MA), and a venous catheter was secured in a left antebrachial vein. First, a 20-min transmission scan was acquired for attenuation correction using three rotating sources of $68 \mathrm{Ge}$. Then regional cerebral blood flow, taken as a marker of local neuronal activity (Jueptner and Weiller, 1995) was estimated during twelve emission scans. Each scan consisted of two frames: a 30-s background frame and a 90-s acquisition frame. The slow intravenous water $\left(\mathrm{H}_{2}{ }^{15} \mathrm{O}\right)$ infusion began $10 \mathrm{~s}$ before the second frame. Six mCi (222 MBq) were injected for each scan, in $5 \mathrm{cc}$ saline, over a period of $20 \mathrm{~s}$. The infusion was totally automated in order not to disturb the subject during the scanning period. Data were reconstructed using a Hanning filter (cutoff frequency: 0.5 cycle/pixel) and corrected for attenuation and background activity. The cognitive tasks were randomly distributed between subjects, with the exception that a cognitive task was not administered twice in succession.

\section{Cognitive Tasks}

Subjects performed a modified version of the Hayling task initially described by Burgess and Shallice (1996). This modification was necessary to make the test suitable for the PET activation procedure. The material consisted of a set of 270 sentences. These sentences were selected from a pool of 500 sentences on the basis of pretests in a group of 21 subjects, which confirmed that the last word of each sentence could be predicted easily from the context of the sentence with a particularly high probability (the last word had to be produced in less than three seconds and words which induced more than five responses other than the expected one were suppressed). From that set, nine lists of 30 sentences each were selected, matched for the frequency of the last word $[F(8,261)=0.40, P=0.9]$ and the number of words in the sentence $[F(8,261)=1.75, P=0.09]$. Four different conditions were administered: (1) Rest: subjects lay with their eyes closed during the prescribed period. (2) Reading: subjects were required to read aloud the last word of visually presented sentences. A trial was considered as correct when the last word was correctly read. (3) Response initiation: subjects were required to provide an appropriate word to complete visually presented sentences from which the last word was missing (e.g., "The captain wanted to stay with the sinking SHIP"). A trial was considered as incorrect when the response was not that predicted from the results of the pretests. (4) Response inhibition: subjects were instructed to provide a word which made no sense at all in the context of a visually presented sentence from which the last word was missing (e.g., "Most cats see very well at BANANA"). From the subjects' responses, an error score was calculated which was a measure of the overall semantic related-ness of each response to its corresponding sentence. Three points were given if the word was a straightforward completion of the sentence, and 1 point for a word that was semantically related to the sentence in some way. No point was given when responses were unrelated to the sentence.

Sentences (48 point Helvetica font) were presented one at a time on a visual display and subjects responded aloud. Sentences remained on the screen until the subject gave a response (self-paced procedure) and each new sentence was presented $1500 \mathrm{~ms}$ after the response was given. Subjects' responses were recorded. Subjects were 
trained on each cognitive task before the PET session. More precisely, in the inhibition condition, subjects were administered a series of sentences and responses in order to indicate what a correct response was (e.g., a response not semantically related to the remainder of the sentence or not phonologically related to the word to be inhibited). Examples of strategies leading to a correct response but involving few inhibition processes (e.g., to name an object in the room or to use systematically the word to inhibit in the previous sentence as a response) were also presented and subjects were asked to avoid such strategies. This training phase was undertaken in order to try to elicit similar cognitive (inhibitory) processes in all subjects. Although such training could induce cognitive processes slightly different from those explored with the classical version of the Hayling task, it seems really important to undertake that training in order to reduce the use of cognitive strategies other than inhibitory to perform the task. After the scanning session, post-hoc questioning of the subjects indicated that they had perfectly complied with the task instructions. Three minutes before each acquisition, the instructions were repeated. Each of the four conditions were performed three times during the session and were counterbalanced between subjects to control for order effects.

\section{Data Analysis}

Data were analyzed using statistical parametric mapping (SPM99; Welcome Department of Cognitive Neurology, Institute of Neurology, London, UK) implemented in MATLAB. For each subject, all scans were realigned together, then normalized to a standard PET template using the same transformations (Frackowiak et al., 1997). Finally, PET images were smoothed using a Gaussian kernel of $16 \mathrm{~mm}$ full width at half maximum to accommodate intersubject differences in gyral and functional anatomy and to suppress high frequency noise in the images. Such transformations of the data allow for voxel by voxel averaging of data across subjects and for direct cross-reference to the anatomical features in the standard stereotactic atlas (Talairach and Tournoux, 1988).

Differences in global activity within and between subjects were removed by analysis of covariance on a voxel by voxel basis with global count as covariate and regional activity across subjects for each task as treatment (Friston et al., 1990). The across-task comparisons were first performed by averaging between paired measurements (fixed effect model). For each voxel in stereotactic space, the analysis of covariance (AN-COVA) generated a condition-specific adjusted mean $\mathrm{rCBF}$ value (normalized to $50 \mathrm{ml} / 100 \mathrm{ml} / \mathrm{min}$ ) and an associated adjusted error variance. The ANCOVA allowed the comparison of the means across conditions on a voxel-by-voxel basis using the $t$ statistic. The resulting sets of $t$ values constituted statistical parametric maps [SPM $(t)]$ (Friston et al., 1991). The $\operatorname{SPM}(t)$ were transformed to the unit normal distribution $[\operatorname{SPM}(Z)]$. The design of our study was a cognitive subtraction paradigm. In this design, the reading task was compared to the rest in order to determine the cerebral areas associated with the processing of a sentence and the production of a verbal response. The initiation task was compared to the reading task in order to determine the cerebral areas associated to the generation of a response highly constrained by the context. Finally, the inhibition task was compared to the initiation task to determine the cerebral areas involved in the inhibition process of a predominant response. This last comparison was also performed using a random effect model (Holmes and Friston, 1998), in order to specify cerebral areas involved in inhibition processes which were activated in each of our twelve subjects. The random effect model is based on the approach of mean summary statistics on repeated measures (Friston and Pocock, 1992). This type of analysis is usually used in neuroimaging studies to accommodate both interindividual and intraindividual variability of PET data and requires that all members of the population show this effect such that its expectation is greater than under the null hypothesis.

A SPM thresholded at $P<0.001$ (voxel level) was used, with further correction for multiple comparisons $(P<$ 0.05). SPM was thresholded to $P<0.001$ (uncorrected for multiple comparisons), when looking for activation in a region predicted from previous studies. 
TABLE 1: Comparison of the Reading Condition to the Rest Condition

Stereotactic coordinates

\begin{tabular}{|c|c|c|c|c|}
\hline \multirow[b]{2}{*}{ Brain area } & & & & \multirow[b]{2}{*}{$T$ value } \\
\hline & $x$ & $y$ & $z$ & \\
\hline \multicolumn{5}{|c|}{$P$ value $<0.001$, with further corrections for multiples comparisons } \\
\hline L inferior occipital gyrus (BA 18) & -24 & -96 & -6 & 12.64 \\
\hline L lingual gyrus (BA 18) & -4 & -78 & 6 & 12.49 \\
\hline L striate gyrus (BA 17) & -14 & -84 & 6 & 12.40 \\
\hline \multirow[t]{4}{*}{ L and R precentral gyrus (BA 6) } & -52 & -8 & 42 & 9.78 \\
\hline & -60 & 0 & 20 & 5.50 \\
\hline & 52 & -4 & 42 & 6.64 \\
\hline & 58 & -4 & 36 & 6.47 \\
\hline Supplementary motor area (BA 6) & 2 & 4 & 56 & 6.21 \\
\hline \multirow{4}{*}{$\begin{array}{l}\text { L middle and superior temporal gyrus } \\
\text { (BA 21/22) }\end{array}$} & -68 & -26 & 0 & 6.18 \\
\hline & -60 & 36 & 0 & 5.26 \\
\hline & -54 & 6 & -6 & 5.49 \\
\hline & -58 & -48 & 6 & 5.27 \\
\hline \multirow[t]{3}{*}{ L and $\mathrm{R}$ superior parietal gyrus (BA 7) } & -28 & -60 & 48 & 6.47 \\
\hline & 32 & -64 & 46 & 5.08 \\
\hline & 32 & -60 & 46 & 4.84 \\
\hline
\end{tabular}

Note. Coordinates and $T$ scores for voxels in which there was significant activation foci when reading task was compared to rest. L, left hemisphere; $\mathrm{R}$, right hemisphere. $x, y, z$ (in $\mathrm{mm}$ ) refer to coordinates in the Talairach space (Talairach and Tournoux, 1988).

\section{RESULTS}

\section{Neuropsychological Performances}

The response times for each condition (in milliseconds, mean \pm SD) were the following: reading: $1965 \pm 442$; initiation: $2273 \pm 542$; inhibition: $4760 \pm 1450$. Analysis of variance showed that the reactions times were different between the three conditions $[F(2,20)=57, P<0.0001] .{ }^{1}$ The error score in the reading and initiation conditions (defined as the number of times a subject gave a response other than that expected) were very low $(0.03 \pm 0.1$ and $0.58 \pm 0.54$, respectively), while the overall semantic relatedness in the inhibition condition was $15.49 \pm 2.87$.

\section{Imaging Data}

Reading-Rest. Increases of $\mathrm{rCBF}$ in the reading task in comparison to the rest condition were observed in the left inferior occipital and lingual gyrus (BA 18), as well as in the left striate gyrus (BA 17). Foci of activation were also found in the right and left precen-tral gyrus (BA 6), supplementary motor area (BA 6), left middle and superior temporal gyrus (BA 21/22) as well as in the superior parietal bilaterally (BA 7) (Table 1 and Fig. 1A).

Initiation-Reading. The comparison of the initiation condition to the reading condition showed increases of activity in the left frontal operculum (BA 45/47; uncorrected level, $P<0.001$ ). That area was previously found in the study of Nathaniel-James et al. (1997; voxel coordinates: $x=-54, y=20, z=4)$. Moreover, when a conjunction analysis was performed between the contrasts "initiation-reading" and "inhibition-rest" (in order to emphasize cerebral areas specifically devoted to the association of an automatic semantic response to a sentence), a similar cerebral area was found to be significantly activated (corrected level, $P<0.001$ ) (Table $2 \mathrm{a}$,

\footnotetext{
1 The outcome of significantly different reaction times between the three conditions is that different numbers of sentences are being processed during the scanning period. Therefore the conditions are not matched for perceptual input and motor response. In order to asses the influence of these factors on cerebral activity, the comparison of the different conditions was also performed with the number of sentences processed as a confound covariable. This analysis yielded similar results to that reported in Tables 1, 2, and 3.
} 
$2 \mathrm{~b}$ and Fig. 1B).

Inhibition-Initiation. When the inhibition task was compared to the initiation task, increases of activity were found in the middle frontal gyrus (left: BA 9 and BA 46; bilaterally: BA 10) and in the anterior part of the inferior frontal gyrus (BA 47, bilaterally). Activation foci were also observed in the left inferior frontal gyrus (BA 45) and in the left orbitofrontal gyrus (BA 11) (Table 3 and Fig. 1C). In order to allow generalization of these results to the general population, random effect analysis was performed and this demonstrated left-sided increases of activity in the middle frontal gyrus (BA 9 and BA 10) and inferior frontal gyrus (BA 45). Moreover, plots of activity in these regions were determined for the different conditions (Fig. 2). These plots indicate that cerebral activity in left BA 45 is significantly higher in the inhibition condition than in all other tasks. Activity in the left middle frontal gyrus (BA 9 and BA 10) is increased during the inhibition task, while it is relatively decreased in the initiation condition.

Correlation analyses. In order to determine the cerebral areas most involved in initiation and inhibition processes, correlation analysis between response times and cerebral metabolism for each of these conditions were computed on a voxel-by-voxel basis. Statistical analysis contrasted the correlations obtained for each condition in order to isolate cerebral areas specifically devoted to initiation processes on the one hand and inhibition processes on the other. Results showed that the speed of response in the initiation condition is specifically and positively correlated to the cerebral metabolism in the left and right inferior parietal gyrus (BA 40 ) and in the left superior temporal gyrus (BA 22). Otherwise, speed of response in the inhibition condition is specifically and positively correlated to the cerebral metabolism in the middle frontal gyrus (BA 10 and BA 8/9) bilaterally, in the medial frontal gyrus (BA 6/8), in the left superior parietal (BA 7) and in the left cuneus (BA 19) (Table 4).

\section{DISCUSSION}

The aim of the present study was to reexamine the cerebral areas related to the inhibition processes involved in the Hayling task (Burgess and Shallice, 1996), that is to say the processes which allow restraining access of strong but situationally inappropriate responses. More specifically, changes of cerebral activity were assessed in several conditions elaborated from the original Hayling task and permitting to clearly dissociate the inhibition component from the initiation of a response: rest, reading the last word of a sentence, production of a word which completes a sentence and which is highly constrained by the context of the sentence (initiation condition), and production of a word that completes a sentence but which makes no sense in the context of the sentence (inhibition condition: inhibition of a word predictable by the remainder of the sentence).

When the cerebral activity in the read condition was contrasted to that found during the rest condition, activation foci were detected in the inferior occipital region, inferior and middle temporal areas as well as in the superior parietal region. Foci of activation were also found in the precentral gyrus and supplementary motor area. Comparing cerebral areas involved in reading aloud and silently, Price et al. (1996) concluded that the fusiform and lingual gyrus are involved in the visual analysis of the items, while the precentral gyrus and supplementary motor area are involved in the articulatory processes during the production of the response. They also suggested that the activity in the right temporal area could be attributed to the auditory processing of the verbal response, since an activation of this area was found during the reading aloud condition only.

The comparison of the initiation condition and the reading condition showed increases of activity in the left inferior frontal gyrus (BA 45/47) and the involvement of these areas in initiation processes was con- firmed by a conjunction analysis between the contrasts "initiation-reading" and "inhibition-rest." Activity in BA 45 was previously found in the Nathaniel-James et al. (1997) study. Moreover, in the present study, the response times in the initiation condition were positively correlated to the left superior temporal gyrus (BA 22) and the inferior parietal (BA 40) bilaterally. These cortical areas would appear to be areas for the storage of semantic information. Indeed, increases of $\mathrm{rCBF}$ were found in the superior temporal area when cerebral activity was compared between a semantic decision task and a phonological segmentation task, both tasks being performed on the same words (Price et al., 1997). Otherwise, the increase of activity in the inferior frontal gyrus (BA 45/47) during the initiation condition was close to cerebral areas activated during semantic classification and generation tasks (Demb et al., 1995; Thompson-Schills et al., 1997; for a review, see Cabeza and Nyberg, 2000). In agreement with these studies, we can suggest that left BA 45/47 is involved in generic semantic retrieval operations, such as the selection and evaluation of a semantic response. Surprisingly, changes in activity are relatively small when the initiation task is compared to reading. One possible explanation is that in both conditions, sentences with a highly predictable ending were used. Thus, in the reading condition, subjects would have direct access to the end the sentence before reading it. Finally, when the inhibition condition was compared to the initiation condition, increases of activity were found bilaterally in the middle frontal gyrus (left: BA 9, BA 10, and BA 46; right: BA 10), in the inferior frontal gyrus (left: BA 45 and BA 47; right: BA 47) and in the left 
orbitofrontal cortex (BA 11). Changes in activity between the two conditions that are common to all subjects (and thus can be generalized to the population) were localized in left-sided middle (BA 10 and BA 9) and inferior (BA 45) frontal areas. Moreover, the response times were correlated with the anterior left and right prefrontal cortex (BA 10, BA 8/9, BA 6/8), with the left superior parietal cortex (BA 7) and with the cuneus (BA 19). These results are in agreement with the hypothesis of an implication of the prefrontal areas in inhibitory control processes and clearly differ from those obtained by Nathaniel-James et al. (1997), who did not observe a prefrontal activity when they contrasted the initiation and inhibition conditions of the Hayling task. However, in the Nathaniel-James et al. study, the sentences were presented every $6 \mathrm{~s}$ and not directly after the production of a response. Thus, subjects had some supplementary time in each condition during which cognitive processes were not controlled, and consequently, this could have made the task less sensitive to inhibition processes. It could be argued that the discrepancy of results between Nathaniel-James et al. and the present study is due to the fact that the instructions given to subjects before the inhibition task were different. However, this seems unlikely since more activation was observed in prefrontal areas despite our instructions, which reduced the use of cognitive processes other than inhibition.

Our study showed that several cerebral areas are involved during the inhibition condition of the Hayling task. In a sense, this is not surprising since this condition involves, in comparison to the initiation condition, different types of processes, not only inhibition of a prevalent response but also lexical access, search strategies and working memory at least. The cerebral areas related to these processes have been explored in a series of studies. Therefore, the cerebral areas associated to the inhibition condition of the Hayling task can be discussed in relation to the results obtained in these previous studies.

Among these regions, the inferior frontal area (BA 45/47) is already activated when subjects have to initiate a response highly constrained by the context (as indicated by the comparison of the initiation and reading conditions) and, in agreement with previous studies, we attributed to that region a role in the selection and evaluation of a semantic response (Thompson-Schills et al., 1997). However, the activation of the inferior frontal area in the inhibition condition was larger, bilateral, and more anterior, which is also consistent with our previous interpretation, suggesting an involvement of that region in selection and evaluation of a semantic response. Indeed, both the initiation and inhibition condition require the evaluation of the ap- propriateness of the provided response in comparison to the expected response. This matching process is more important in the inhibition condition than in the initiation condition since, in the latter, a single correct response exists while, in the former, subjects have to choose among a set of correct responses the one that is the least related to the sentence. This anterior versus posterior distribution of ventrolateral prefrontal activation might correspond to the selection of a semantic response from among numerous (relative to few) alternatives (Gabrieli et al., 1998; see also de Zubicaray et al., 2000). ${ }^{2}$

Increase of activity observed during the inhibition condition in the left middle frontal gyrus (BA 9) has already been described in various tasks, requiring an important planning of the required response (e.g., Baker et al., 1996; Dagher et al., 1999; Owen et al., 1996) or an active manipulation of information stored in working memory (Collette et al., 1999). On the basis of these studies, the involvement of that left middle frontal area during the inhibition of a prevalent semantic response could be attributed to the manipulation of information in order to build up an efficient research strategy and choose between different alternatives the word the least related to the context of the sentence (see also de Zubicaray et al., 2000 for a globally similar interpretation of the role of that area in semantic inhibition processes).

Finally, foci of activation were found in a lower left middle frontal region (BA 10). One general process attributed to that area concerns the evaluation and selection of internally generated information (Christoff and Gabrielli, 2000). Activity in that region was also described during updating tasks in which subjects had to remember serially the (four or six) last items of lists of unknown length (Salmon et al., 1996; Van der Linden et $a l ., 1999)$. The function of this updating process is to modify continuously the content of working memory according to newer external (sensory input) or internal (long-term memory retrieval) information. That area was also found activated in studies using the Stroop inhibition paradigm (George et al., 1994; Taylor et al., 1997) and during the realization of go/no-go tasks (Garavan and Stein, 1999). Taken as a whole, the results of these studies are in agreement with the hypothesis of an involvement of BA 10 in inhibition processes. Indeed, both the Stroop and the go/no-go tasks require the inhibition of a prevalent response, while the updating task requires to actively suppress the no-longer relevant items. Moreover, in the present study, activity in that region was correlated with

\footnotetext{
2 It is important to indicate that other interpretations have been proposed concerning the role of this area in cognition. Nathaniel-James et al. (1997) consider it to be related to the generation of words in the absence of an external cue. Another view suggests the ventral prefrontal involvement in the active retrieval and reproduction of stored information in working memory (Owen et al., 1999). In that context, that area could be involved in the executive processes necessary to the matching between the sentence maintained in working memory and the result in the search for a non-related response in the posterior semantic regions. Finally, Konishi et al. (1999) attributed to that region a specific role in inhibition processes.
} 
the time necessary to find a response unrelated to the context of the sentences. Consequently, we could argue that the region underlies (at least) semantic inhibitory processes involved in the inhibition condition of the Hayling task.

A general problem related to the exploration of the neural substrates of inhibitory processes concerns the lack of specification of the concept of inhibition. Indeed, this concept cannot be considered as unitary and refers probably to very different mechanisms (Connely and Hasher, 1993; McDowd, 1997) and the exact relationships between these mechanisms are not clearly established (Stolzfus et al., 1993; Kieley and Hartley, 1997). In that context, the aim of future studies will be to determine whether some inhibition processes are common to different tasks (as attested by similar neural substrates) or whether the various mechanisms of inhibition are linked to distinct cerebral areas. At present, only one study has tackled this question. Ko-nishi et al. (1999) showed a common focus of activity in the right inferior prefrontal area (BA 45/44), which they associated to inhibition processes for different kinds of material. However, the tasks used (the go/ no-go and WCST) share other cognitive characteristics (i.e., working memory, ...) than the necessity to inhibit current action schemata, and so the inferior pre-frontal activity could be also related to these processes.

In conclusion, the present study has confirmed the role of the left middle (BA 9 and BA 10) and inferior (BA 45) prefrontal areas in semantic inhibitory processes involved in the Hayling task. The contribution of several prefrontal areas during the inhibition condition of this task is not surprising since this is a complex task involving other cognitive processes than inhibition, such as planning, semantic search, manipulation of information, selection and evaluation of the response. In order to better dissociate inhibition from other cognitive processes, future studies should be based on simpler tasks (see Chee et al., 2000, for an example of such a task).

A
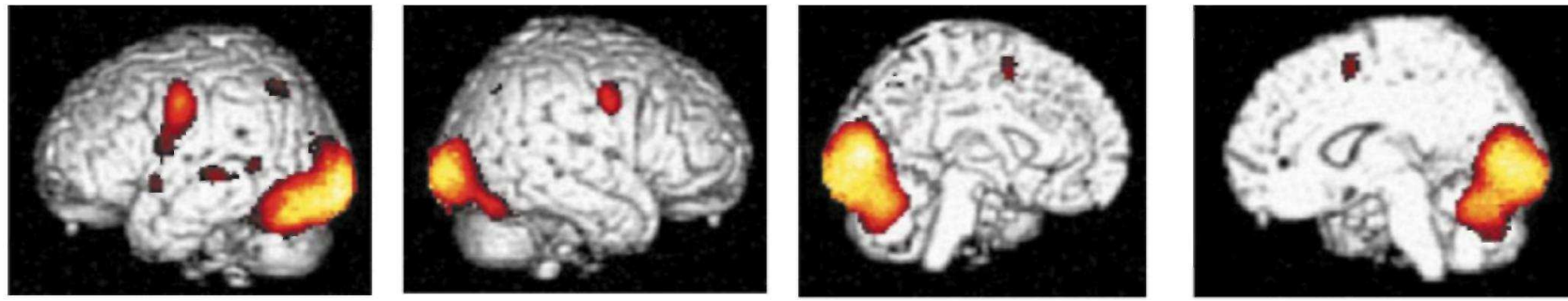

B
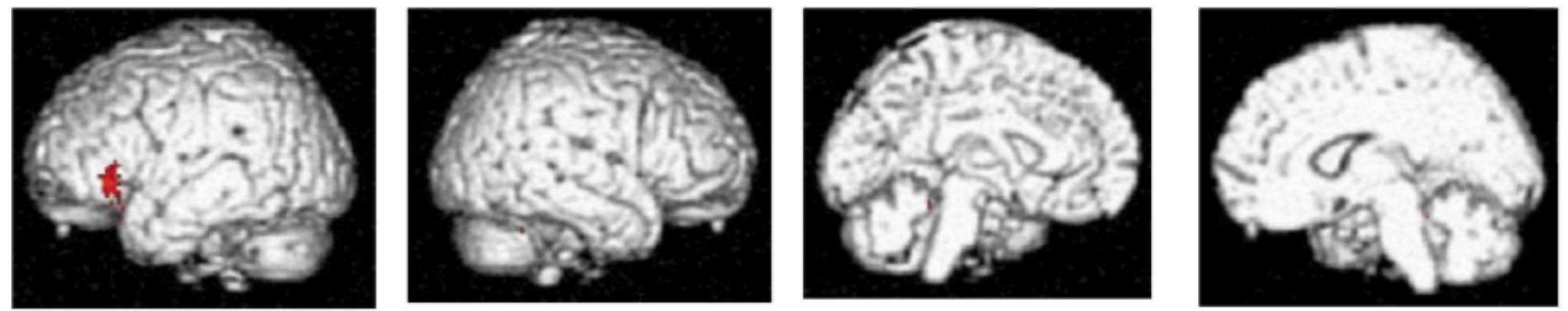

$\mathrm{C}$
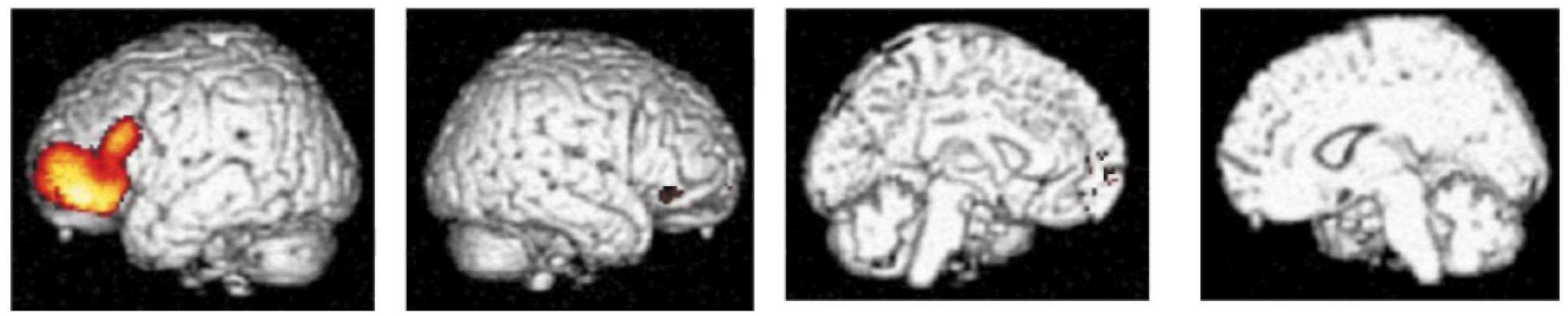

FIG. 1. Brain activation (corrected for multiple comparisons) observed (A) during reading compared to rest; (B) during initiation compared to reading; (C) during inhibition compared to initiation. Coordinates of all significant regions are given respectively in Tables 1-3. Brain areas are rendered on a standard brain conforming to stereotactic space. 
TABLE 2a: Comparison of the Initiation Condition to the Reading Condition

Stereotactic coordinates

Brain area

$x \quad y \quad z$

$T$ value

$P$ value $<0.001$, uncorrected for multiples comparisons

L inferior frontal gyrus (BA 45/47)

$\begin{array}{llll}-52 & 22 & -18 & 3.69 \\ -54 & 24 & -6 & 3.63 \\ -56 & 26 & 4 & 3.38\end{array}$

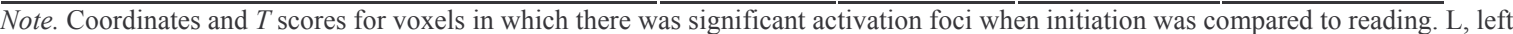
hemisphere; R, right hemisphere, $x, y, z$ (in mm) refer to coordinates in the Talairach space (Talairach and Tournoux, 1988).

TABLE 2b: Conjunction Analysis between the Contrasts "Initiation-Reading” and "Inhibition-Rest"

Brain area

Stereotactic coordinates

$T$ value

$\begin{array}{lll}x & y\end{array}$

$P$ value $<0.001$, with further comparisons for multiples comparisons

L inferior frontal gyrus (BA 45/47)

$\begin{array}{llll}-52 & 22 & -18 & 3.69 \\ -54 & 24 & -6 & 3.63 \\ -56 & 26 & 4 & 3.38\end{array}$

Note. Coordinates and $T$ scores for voxels in which there was significant conjoint activation foci when initiation and inhibition tasks were compared to reading and rest. $\mathrm{L}$, left hemisphere; $\mathrm{R}$, right hemisphere, $x, y, z$ (in $\mathrm{mm}$ ) refer to coordinates in the Talairach space (Talairach and Tournoux, 1988) 
TABLE 3: Comparison of the Inhibition Condition to the Initiation Condition

Brain area Stereotactic coordinates

$T$ value

$\begin{array}{lll}x & y & z\end{array}$

Fixed effect. $P$ value $<0.001$, with further corrections for multiples comparisons

L middle frontal gyrus (BA 9)

$\begin{array}{lll}-32 & 20 & 32\end{array}$

$-44$

22

22

L middle frontal gyrus (BA 46)

$-52 \quad 22$

26

Lidie frontal gyins (BA 46)

$-54$

30

26

4.96

$-40$

38

18

4.94

$-46$

40

10

5.66

L middle frontal gyrus (BA 10)

$$
-42
$$

46

$-4$

7.85

$-36$

50

10

6.54

$-34$

58

4

6.86

$-30$

64

10

6.36

$\mathrm{R}$ middle frontal gyrus (BA 10)

38

66

$-2$

4.73

$\mathrm{L}$ inferior frontal gyrus (BA 45)

$-44$

24

2

5.46

$-50$

34

4

5.31

L inferior frontal gyrus (BA 47)

$$
-36
$$

26

$-8$

5.90

$$
-50
$$

$-2$

$-44$

$-8$

7.46

$\mathrm{R}$ inferior frontal gyrus (BA 47)

$$
60
$$

42

$-10$

6.39

L orbitofrontal gyrus (BA 11)

$-28$

$-20$

Random effect. $P$ value $<0.001$, corrected for multiples comparisons

L middle frontal gyrus (BA 9)

$$
-32
$$

16

$$
30
$$

14.52

L middle frontal gyrus (BA 10)

$\begin{array}{ll}-38 & 44\end{array}$

$-4$

13.59

$\mathrm{L}$ inferior frontal gyrus (BA 45)

$-52 \quad 20$

18

14.80

$-44$

20

22

12.13

Note. Coordinates and $T$ scores for voxels in which there was significant activation foci when inhibition task was compared to initiation task. $\mathrm{L}$, left hemisphere; $\mathrm{R}$, right hemisphere. $x, y, z$ (in $\mathrm{mm}$ ) refer to coordinates in the Talairach space (Talairach and Tournoux, 1988). 

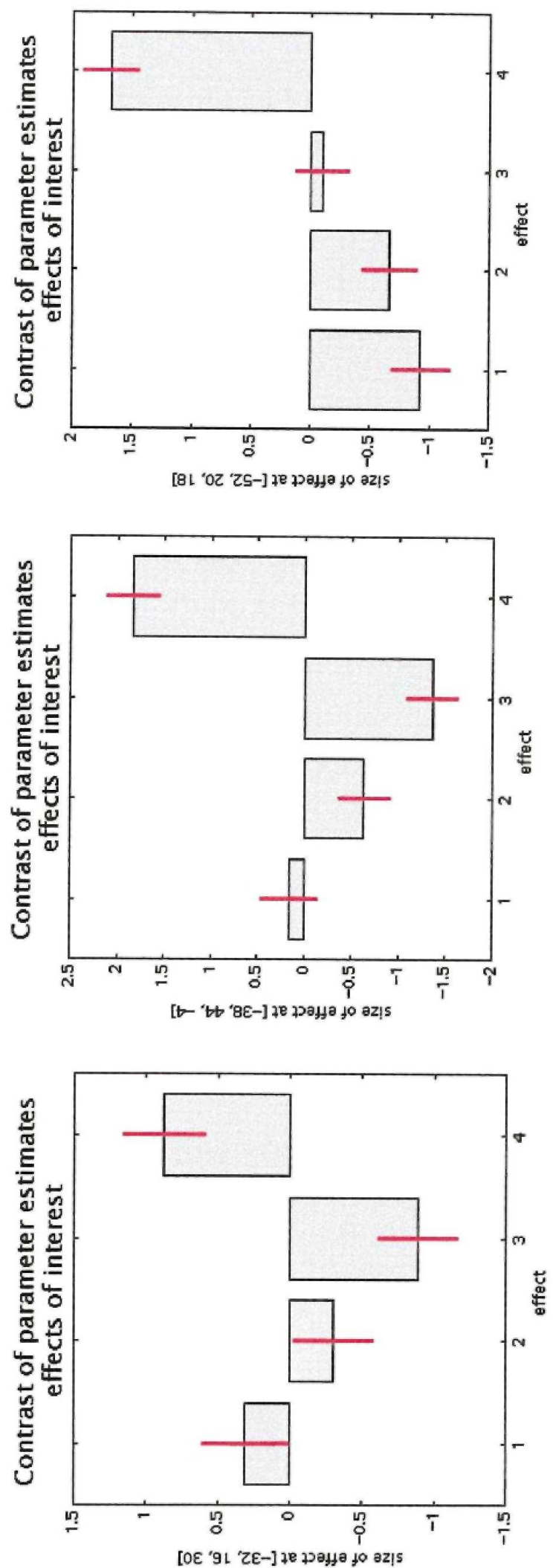

FIG. 2. Plots of relative cerebral activity in the four cognitive tasks for the voxels significantly more activated in the inhibition condition than in the initiation condition (random effect analysis, corrected $P$ value $<0.001$ ). Coordinates of each voxel are indicated on the $\mathrm{Y}$ axis and cognitive tasks are represented on the $\mathrm{X}$ axis (1, rest; 2, reading; 3 , initiation; 4 , inhibiti 
TABLE 4 : Correlation Analyses

Brain area

$\begin{array}{lll}x & y\end{array}$

$P$ value $<0.001$, with further corrections for multiples comparisons

Correlation between speed of response and cerebral metabolism in the initiation condition

$\begin{array}{lllll}\text { R inferior parietal (BA 40) } & 56 & -28 & 20 & 6.67 \\ & 60 & -30 & 28 & 6.58 \\ & 58 & -12 & 8 & 6.57 \\ \text { R superior parietal (BA 7) } & 16 & -58 & -58 & 4.97 \\ & 18 & -66 & 54 & 4.38 \\ \text { L superior temporal gyrus (BA 22/42) } & -62 & 20 & 10 & 4.93 \\ & -54 & -4 & -4 & 4.28 \\ & -58 & -26 & 18 & 4.25\end{array}$

Correlation between speed of response and cerebral metabolism in the inhibition condition

L middle frontal gyrus (BA 10)

$\begin{array}{llll}-46 & 44 & -6 & 8.31 \\ -28 & 62 & 0 & 7.93 \\ -28 & 60 & 8 & 8.19\end{array}$

R middle frontal gyrus (BA 10)

$$
32
$$$$
36
$$$$
52
$$$$
-16
$$$$
5.40
$$$$
36 \quad 50
$$$$
-2
$$

R middle frontal gyrus (BA 8/9)

38

$$
62
$$

$$
0
$$

$60 \quad 26$

$54 \quad 22$

Medial frontal gyrus (BA 6/8)

$\begin{array}{ll}-6 & 20\end{array}$

$-4 \quad 32$

$-8 \quad 4$

L superior parietal gyrus (BA 7)

$\begin{array}{llll}-44 & -62 & 48 & 5.06 \\ -52 & -66 & 48 & 4.30 \\ -38 & -64 & 36 & 3.82 \\ 4 & -102 & 22 & 3.51 \\ 0 & -98 & 28 & 4.86 \\ 0 & -94 & 36 & 5.18\end{array}$

Note. Coordinates and $T$ scores for voxels for which there exists a significant $\overline{c o r r e l a t i o n ~ w i t h ~ t h e ~ r e s p o n s e ~ t i m e s ~ i n ~ t h e ~ i n i t i a t i o n ~ a n d ~}$ inhibition conditions. $\mathrm{L}$, left hemisphere; $\mathrm{R}$, right hemisphere, $x, y, z$ (in $\mathrm{mm}$ ) refer to coordinates in the Talairach space (Talairach and Tournoux, 1988).

\section{ACKNOWLEDGMENTS}

This work was supported by the Belgian National Fund for Scientific Research (FNRS), the "Fondation Médicale Reine Elisabeth," and the Interuniversity Pole of Attraction Program P4/22, Belgian State, Prime Minister's Office, Federal Office for Scientific, Technical and Cultural Affairs. F. Collette is a Postdoctoral Researcher at the FNRS. 
Published in: Neuroimage (2001), vol. 14, iss. 2, pp. 258-267

Status: Postprint (Author's version)

\section{REFERENCES}

Ahola, K., Vilkki, J., and Servo, A. 1996. Frontal tests do not detect frontal infarctions after ruptured intracranial aneurysm. Brain Cogn. 31: $1-16$.

Anderson, S. W., Damasio, H., Jones, R. D., and Tranel, D. 1991. Wisconsin card sorting test performance as a measure of frontal lobe damage. J. Clin. Exp. Neuropsychol. 13: 909-922.

Andrès, P., and Van der Linden, M. 2000. Supervisory Attentional System in patients with focal frontal lesions. J. Clin. Exp. Neuro-psychol., in press.

Baddeley, A. D. 1986. Working Memory. Clarendon Press, Oxford.

Baker, S. C., Rogers, R. D., Owen, A. M., Frith, C. D., Dolan, J. R., Frackowiak, R. S. J., and Robbins, T. W. 1996. Neural systems engaged by planning: A PET study of the Tower of London task. Neuropsychologia 34: 515-526.

Bench, C. J., Frith, C. D., Grasby, P. M., Friston, K. J., Paulesu, E., Frackowiak, R. S. J., and Dolan, R. J. 1993. Investigations of the functional anatomy of attention using the Stroop test. Neuropsy-chologia 31: 907-922.

Burgess, P. W., and Shallice, T. 1996. Response suppression, initiation and strategy use following frontal lobe lesions. Neuropsycho-logia 34: $263-273$.

Bush, G., Whalen, P. J., Rosen, B. R., Jenike, M. A., McInerney, S. C., and Rauch, S. L. 1998. The counting stroop: An interference task specialized for functional neuroimaging. Validation study with functional MRI. Hum. Brain Mapp. 6: $270-282$.

Cabeza, R., and Nyberg, L. 2000. Imaging cognition II: An empirical review of 275 PET and fMRI studies. J. Cogn. Neurosci. 12 : 1-47.

Chee, M. W. L., Sriram, N., Siong Soon, C., and Ming Lee, K. 2000. Dorsolateral prefrontal cortex and the implicit association of concepts and attributes. Neuroreport 11: 135-140.

Collette, F., Salmon, E., Van der Linden, M., Chicherio, C., Belleville, S., Degueldre, C., Delfiore, G., and Franck, G. 1999. Regional brain activity during tasks devoted to the central executive of working memory. Cogn. Brain Res. 7: 411-417.

Connelly, L., and Hasher, L. 1993. Aging and the inhibition of spatial location. J. Exp. Psychol. Hum. Percept. Perform. 19: 1238-1250.

Christoff, K., and Gabrielli, J. D. E. 2000. The frontopolar cortex and human cognition: Evidence for a rostrocaudal hierarchical organization within the human prefrontal cortex. Psychobiology 28: 168- 186.

Dagher, A., Owen, A. M., Boecker, H., and Brooks, D. J. 1999. Mapping the network for planning: A correlational PET activation study with the Tower of London task. Brain 122: 1973-1987.

De Zubicaray, G. I., Zelaya, F. O., Andrew, C., Williams, S. C. R., and Bullmore, E. T. 2000. Cerebral regions associated with verbal response initiation, suppression and strategy use. Neuropsycholo-gia 38: 1292-1304.

Demb, J. B., Desmond, J. E., Wagner, A. D., Vaidya, C. J., Glover, G. H., and Gabrieli, J. D. E. 1995. Semantic encoding and retrieval in the left inferior prefrontal cortex: A functional MRI study of task difficulty and process specificity. J. Neurosci. 15: 5870-5878.

Frackowiak, R., Friston, K., Frith, C., Dolan, R., and Mazziotta, J. C. 1997. Human Brain Function. Academic Press, London.

Friston, K. J., Frith, C. D., Liddle, P. F., and Frackowiak, R. S. J. 1991. Comparing functional (PET) images: The assessment of significant changes. J. Cereb. Blood Flow Metab. 11: 690-699.

Friston, K. J., Frith, C. D., Liddle, P. F., Dolan, R. J., Lammertsma, A. A., and Frackowiak, R. S. J. 1990. The relationship between global and local changes in PET scans. J. Cereb. Blood Flow Metab. 10: 458-466.

Friston, K. J., Holmes, A. P., Worsley, K. J., Poline, J.-B., Frith, C. D., and Frackowiak, R. S. J. 1995. Statistical parametric maps in functional imaging: A general linear approach. Hum. Brain Mapp. 2: 189-210.

Friston, K. J., and Pocock, S. J. 1992. Repeated measures in clinical trials: An analysis using mean summary statistics and its implication for design. Statist. Med. 11: 1685-1704.

Gabrieli, J. D. E., Poldrack, R. A., and Desmond, J. E. 1998. The role of prefrontal cortex in language and memory. Proc. Natl. Acad. Sci. USA 95: 906-913.

Garavan, H., and Stein, E. A. 1999. Right hemispheric dominance of inhibitory control: An event-related functional MRI study. Proc. Natl. Acad. Sci. USA 96: 8301-8306.

George, M. S., Ketter, T. A., Parekh, P. I., Rosinsky, N., Ring, H., Casey, B. J., Trimble, M. R., Horwitz, B., Herscovitch, P., and Post, R. M. 1994. Regional brain activity when selecting a response despite interference: An H2O15 PET study of the Stroop and emotional Stroop. Hum. Brain Mapp. 1: 194-209.

Glosser, G., and Goodglass, H. 1990. Disorders in executive control functions among aphasic patients and other brain-damaged patients. $J$. Clin. Exp. Neuropsychol. 12: 485-501.

Hasher, L., Zacks, R. T., and May, C. P. 1999. Inhibitory control, circadian arousal, and age. In Attention and Performance XVII. Cognitive Regulation of Performance: Interaction of Theory and Application, (D. Gopher and A. Koriat, Eds.), pp. 653-675. MIT Press, Cambridge, MA.

Holmes, A., and Friston, K. 1998. Generalisability, random effects and population inference. Neuroimage 7: 754.

Jueptner, M., and Weiller, C. 1995. Review: Does measurement of regional cerebral blood flow reflect synaptic activity? Implications for PET and fMRI. NeuroImage 2: 148-156.

Kieley, J. M., and Hartley, A. A. 1997. Age-related equivalence of identity suppression in the Stroop color-word task. Psychol. Aging 12: 22-29. 
Konishi, S., Nakajima, K., Uchida, I., Kikyo, H., Kameyama, M., and Miyashita, Y. 1999. Common inhibitory mechanism in human inferior prefrontal cortex revealed by event-related functional MRI. Brain 122: 981-991.

Larrue, V., Celsis, P., Bès, A., and Marc-Vergnes, J.-P. 1994. The functional anatomy of attention in humans: Cerebral blood flow changes induced by reading, naming and the Stroop effect. J. Cereb. Blood Flow Metab. 14: 958-962.

Leimkhuler, M. E., and Mesulam, M. M. 1985. Reversible go-no go deficits in a case of frontal lobe tumour. Ann. Neurol. 18: 617-619.

Leung, H. C., Skudlarski, P., Gatenby, J. C., Peterson, B. S., and Gore, J. C. 2000. An event-related functional MRI study of the Stroop color word interference task. Cereb. Cortex 10: 552-560.

McDowd, J. M. 1997. Inhibition in attention and aging. J. Gerontol. 52B: P265-P273.

Metzler, C., and Parkin, A. J. 2000. Reversed negative priming following frontal lobe lesions. Neuropsychologia 38: 363-379.

Milner, B. 1964. Some effects of frontal lobectomy in man. In The Frontal Granular Cortex and Behavior (J. M. Warren and K. Akert, Eds.), pp. 313-334. McGraw-Hill, New York.

Mountain, M. A., and Snow-William, W. G. 1993. Wisconsin Card Sorting Test as a measure of frontal lobe pathology: A review. Clin. Neuropsychol. 7: 108-118.

Nathaniel-James, D. A., Fletcher, P., and Frith, C. D. 1997. The functional anatomy of verbal initiation and suppression using the Hayling test. Neuropsychologia 35: 559-566.

Norman, D. A., and Shallice, T. 1986. Attention to action: Willed and automatic control of behavior. In Consciousness and Self Regulation. Advances in Research and Theory (R. J. Davidson, G. E. Schwartz, and D. Shapiro, Eds.), pp. 1-18. Plenum Press, New York.

Owen, A. M., Doyon, J., Petrides, M., and Evans, A. C. 1996. Planning and spatial working memory: A positron emission tomography study in humans. Eur. J. Neurosci. 8: 353-364.

Owen, A. M., Herrod, N. J., Menon, D. K., Clark, J. C., Downey, S. P. M. J., Carpenter, T. A., Minhas, P. S., Turkheimer, F. E., Williams, E. J., Robbins, T. W., Sahakian, B. J., Petrides, M., and

Pickard, J. D. 1999. Redefining the functional organization of working memory processes within human lateral prefrontal cortex. Eur. $J$. Neurosci. 11: 567-574.

Pardo, J. V., Pardo, P. J., Janer, K. W., and Raichle, M. E. 1990. The anterior cingulate cortex mediates processing selection in the Stroop attentional conflict paradigm. Proc. Natl. Acad. Sci. USA 87: 256-259.

Perret, E. 1974. The left frontal lobe of man and the suppression of habitual responses in verbal categorical behavior. Neuropsycholo-gia 12 : $323-330$

Price, C. J., Moore, C. J., Humphreys, G. W., and Wise, R. J. S. 1997. Segregating semantic from phonological processes during reading. J. Cogn. Neurosci. 9: 727-733.

Price, C. J., Moore, C. J., and Frackowiak, R. S. J. 1996. The effect of varying the stimulus rate and duration on brain activity during reading. Neuroimage 3: 40-52.

Salmon, E., Van der Linden, M., Collette, F., Delfiore, G., Maquet, P., Degueldre, C., Luxen, A., and Franck, G. 1996. Regional brain activity during working memory tasks. Brain 119: 1617-1625.

Shimamura, A. P., Jurica, P. J., Mangels, J. A., and Gershberg, F. B. 1995. Susceptibility to memory interference effects following frontal lobe damage: Finding from tests of paired-associate learning. J. Cogn. Neurosci. 7: 144-152.

Stoltzfus, E. R., Hasher, L., Zacks, R. T., Ulivi, M. S., and Goldstein, D. 1993. Investigation of inhibition and interference in younger and older adults. J. Gerontol. 48: P179-P188.

Stuss, D. T., Alexander, M. P., Hamer, L., Palumbo, C., Dempster, R., Binns, M., Levine, B., and Izukawa, D. 1998. The effects of focal anterior and posterior brain lesions on verbal fluency. J. Int. Neuropsychol. Soc. 4: 265-278.

Talairach, J., and Tournoux, P. 1988. Co-planar Stereotaxic Atlas of the Human Brain: 3-Dimensional Proportional System: An Approach to Cerebral Imaging. Thieme, Stuttgart.

Taylor, S. F., Kornblum, S., Lauber, E. J., Minoshima, S., and Ko-eppe, R. A. 1997. Isolation of specific interference processing in the Stroop task: PET activation studies. Neuroimage 6: 81-92.

Thompson-Schill, S. L., D’Esposito, M., Aguirre, G. K., and Farah, M. J. 1997. Role of left inferior prefrontal cortex in retrieval of semantic knowledge: A reevaluation. Proc. Natl. Acad. Sci. USA 94: 14792-14797.

Van der Linden, M., Collette, F., Salmon, E., Delfiore, G., Degueldre, C., Luxen, A., and Franck, G. 1999. The neural correlates of updating of information in verbal working memory. Memory 7: 549-560.

Woods, R. P., Cherry, S. R., and Mazziotta, J. C. 1992. Rapid automated algorithm for aligning and reslicing PET images. J. Com-put. Assist. Tomogr. 16: 620-633. 